\title{
Tecnura
}

http://revistas.udistrital.edu.co/ojs/index.php/Tecnura/issue/view/640

DOI: http://dx.doi.org/10.14483/udistrital.jour.tecnura.2014.SE1.a02

INVESTIGACIÓN

\section{Resistencia bajo carga monotónica de mezclas de concreto asfáltico modificadas con gilsonita por vía seca}

\author{
Monotonic loading strength of hot asphalt mixtures modified \\ with gillsonite by dry-way
}

\author{
Hugo Alexander Rondón Quintana*, Edgar Humberto Sánchez Cotte**, \\ Jesús Orlando Castaño Tabares***
}

Citation / Para citar este artículo: Rondón Quintana, H. A., Sánchez Cotte, E. H., \& Castaño Tabares, J. O. (2014). Resistencia bajo carga monotónica de mezclas de concreto asfáltico modificadas con gilsonita por vía seca. Revista Tecnura, Edición especial, 31-37.

Fecha de recepción: 29 de noviembre de 2013 / Fecha de aceptación: 11 de julio de 2014

\section{RESUMEN}

El artículo presenta los resultados experimentales de ensayar tres mezclas asfálticas densas en caliente (concreto asfáltico) tipos MDC-1, MDC-2 y MDC-3 (de acuerdo con las especificaciones del Instituto $\mathrm{Na}$ cional de Vías (INVIAS, 2007) modificadas por vía seca con un asfalto natural de tipo gilsonita (G) proveniente de la región del Cesar, Colombia. Para la elaboración de las mezclas y la evaluación de las mismas se empleó el cemento asfáltico (CA) que actualmente se fabrica en Colombia: CA 60-70 (PG 58-22). Para la evaluación de la resistencia mecánica bajo carga monotónica de las mezclas asfálticas convencionales (sin gilsonita) y modificadas se empleó el ensayo Marshall. Se reporta un incremento notable en la rigidez de las mezclas modificadas cuando se adiciona a las mismas una relación de giIsonita y $\mathrm{CA}$ de $\mathrm{G} / \mathrm{CA}=10 \%$.

Palabras clave: Concreto asfáltico, gilsonita, mezcla asfáltica modificada, resistencia bajo carga monotónica.

\begin{abstract}
This paper presents the experimental results on three hot asphalt mixtures (HMA) types MDC-1, MDC-2 and MDC-3 (INVIAS, 2007) modified by dry-way with a natural asphalt called Gilsonite (G) from Cesar region (Colombia). Asphalt cement AC 60-70 (PG 58-22) was used in the preparation and evaluation of the mixtures, which is currently manufactured in Colombia. For the evaluation of mechanical strength under monotonic loading of conventional (no Gilsonite) and modified asphalt mixtures, the Marshall Test was performed. This paper reports a notable increase in the stiffness of modified mixtures when adding a relationship of Gilsonite and $\mathrm{G} / \mathrm{AC}=10 \%$.
\end{abstract}

Keywords: Asphalt concrete, Gilsonite, modified asphalt mixture, monotonic loading strength.

\footnotetext{
* Ingeniero civil, magíster en ingeniería civil y doctor en ingeniería. Docente, Universidad Distrital Francisco José de Caldas. Bogotá, Colombia. Contacto: harondonq@udistrital.edu.co

** Ingeniero civil, magíster en ingeniería civil con énfasis en gerencia y construcción. Docente, Universidad Distrital Francisco José de Caldas. Bogotá, Colombia. Contacto: esanchezc@udistrital.edu.co

*** Ingeniero civil, máster en ingeniería del hormigón. Docente, Pontificia Universidad Javeriana. Bogotá, Colombia. Contacto: jesus.castano@ javeriana.edu.co
} 


\section{INTRODUCCIÓN}

De acuerdo con (Sagamone, 1995), el asfalto natural es un material bituminoso natural en estado sólido compuesto por hidrocarburos de alto peso molecular en estratos que pueden ir desde algunos centímetros a decenas de metros de espesor; presentan gran variedad en su dureza, por lo que su punto de fusión puede ir de $100{ }^{\circ} \mathrm{C}$ a más de $300{ }^{\circ} \mathrm{C}$. En Colombia existe una gran cantidad de yacimientos de asfaltos naturales. Se identifican depósitos, principalmente en los departamentos de Boyacá, Caquetá, Caldas, Cundinamarca, Tolima, Santander y Cesar.

La gilsonita es uno de los asfaltos naturales de mayor pureza. Posee baja gravedad específica, bajo carbón fijo y contenido de azufre. Este material fue utilizado por uno de los autores para modificar por vía húmeda dos cementos asfálticos colombianos (Rondón \& Reyes, 2012), con el fin de producir una mezcla de concreto asfáltico y evaluar sus propiedades bajo carga monotónica y dinámica. Los resultados obtenidos del estudio demostraron que las mezclas asfálticas modificadas con la gilsonita por vía húmeda (adicionándola al cemento asfáltico) generan mezclas de concreto asfáltico más rígidas, llevando a pensar que tendrían un buen desempeño en climas cálidos. Los valores de estabilidad y rigidez Marshall de las mezclas modificadas fueron mayores para cualquier porcentaje de asfalto y gilsonita, en comparación con la mezcla convencional. Los módulos resilientes de las mezclas modificadas fueron superiores a los alcanzados por las convencionales, y los mayores incrementos se obtuvieron cuando la temperatura del ensayo aumentaba. Lo anterior permitió concluir que la gilsonita como modificador de asfaltos puede ser un material que mejore las características de rigidez y resistencia a las deformaciones permanentes de mezclas que se utilicen en climas cálidos. Una conclusión similar fue reportada por (Liu \& Li, 2008) cuando modificaron un cemento asfáltico de Alaska con gilsonita por vía húmeda en porcentajes entre $3 \%$ y $12 \%$ con respecto al peso total del
CA. (Esfeh, Ghanavati, \& GhaleGolabi, 2011) mencionan que adicionar gilsonita a un asfalto ayuda a incrementar la viscosidad y disminuye la penetración rigidizando el ligante.

En el presente estudio, a diferencia del reportado por (Rondón \& Reyes, 2012), la modificación de las mezclas se ejecutó por vía seca y no por vía húmeda, es decir, la gilsonita se adicionó al agregado pétreo y no al asfalto. Lo anterior, con el fin de medir si las mezclas de concreto asfáltico experimentan un buen comportamiento bajo carga monotónica cuando se modifican con gilsonita por vía seca, ya que esta forma de modificación permite reducir costos de fabricación de las mismas en las plantas de asfalto.

El artículo presenta una fase experimental diseñada con el fin de evaluar la resistencia bajo carga monotónica de tres mezclas de concreto asfáltico de tipos MDC-1, MDC-2 y MDC-3 (de acuerdo con las especificaciones del Instituto Nacional de Vías (INVIAS, 2007) modificadas con un asfalto natural de tipo gilsonita $(G)$ proveniente de la región del Cesar, Colombia. El cemento asfáltico (CA) utilizado para la fabricación de las mezclas es el que actualmente se fabrica en Colombia: CA CA 60-70 (se refiere a un CA cuya medida en el ensayo de penetración ASTM D-5 está en un rango entre 60 y 70 décimas de $\mathrm{mm}$ ). Este ligante presenta un grado de funcionamiento PG 64-22. La gilsonita se adicionó al agregado pétreo de la mezcla asfáltica a alta temperatura (modificación por vía seca). Para la evaluación de la resistencia mecánica bajo carga monotónica de las mezclas asfálticas convencionales (sin aditivo o gilsonita) y modificadas se empleó el ensayo Marshall (INV. E-748-07, AASHTO T 245 - 97).

\section{METODOLOGÍA}

\section{Caracterización de materiales}

En la tabla 1 se presentan los valores obtenidos de los ensayos de caracterización al agregado pétreo. Se observa en esta tabla que los valores de cada 
uno de los ensayos cumplen con el requisito mínimo de calidad exigido por las especificaciones (INVIAS, 2007) para fabricar mezclas de concreto asfáltico de tipos MDC-1, MDC-2 y MDC-3. Para cumplir con las especificaciones del (INVIAS, 2007), se modificó la granulometría original de los agregados, tomando como referencia los valores promedio en porcentajes de la franja granulométrica que exige la especificación para la elaboración de las mezclas (ver la tabla 2). Al cemento asfáltico se le hicieron los ensayos típicos que exige la especificación (INVIAS, 2007) para caracterizarlos y los resultados se presentan en la tabla 3. La giIsonita utilizada proviene de la mina San Alberto (Cesar, Colombia). Una descripción detallada de la gilsonita puede consultarse en (INVIAS, 1997). Este material presenta un peso específico de 1.10 $\mathrm{g} / \mathrm{cm}^{3}$ y partículas de coloración negra brillante que pasan el tamiz No. 40 en un ensayo de granulometría por tamizado (ver la figura 1). Sobre el CA convencional y modificado se efectuaron ensayos de penetración (INV. E-706) a diferentes temperaturas y punto de ablandamiento, siguiendo los procedimientos recomendados por (INVIAS, 2013a).

Tabla 1. Caracterización del agregado pétreo y granulometría.

\begin{tabular}{llc}
\hline \multicolumn{1}{c}{ Ensayo } & \multicolumn{1}{c}{ Método } & Resultado \\
\hline Peso específico (gruesos y finos) & ASTM D 854-00 & 2,62 \\
\hline Equivalente de arena & ASTM D 2419-95 & $76 \%$ \\
\hline Caras fracturadas & ASTM D 5821-01 & $87 \%$ \\
\hline Índice de alargamiento & NLT 354-91 & $9,5 \%$ \\
\hline Índice de aplanamiento & NLT 354-91 & $9,5 \%$ \\
\hline Ataque en sulfato de sodio & ASTM C 88-99a & $12,9 \%$ \\
\hline Microdeval & ASTM D6928-03 & $22,3 \%$ \\
\hline Resistencia al dessgaste & ASTM C 131-01 & $24,6 \%$ \\
\hline
\end{tabular}

Fuente: Elaboración propia.

Tabla 2. Granulometría de agregados pétreos para la fabricación de las mezclas.

\begin{tabular}{lllll}
\hline \multicolumn{2}{c}{ Tamiz } & \multicolumn{3}{c}{ Mezcla } \\
\hline Normal & Alterno & MDC-1 & MDC-2 & MDC-3 \\
\hline $37.5 \mathrm{~mm}$ & $11 / 2^{\prime \prime}$ & - & - & - \\
\hline $25.0 \mathrm{~mm}$ & $1^{\prime \prime}$ & 100 & - & - \\
\hline $19.0 \mathrm{~mm}$ & $3 / 4^{\prime \prime}$ & $80-95$ & 100 & - \\
\hline $12.5 \mathrm{~mm}$ & $1 / 2^{\prime \prime}$ & $67-85$ & $80-95$ & - \\
\hline $9.5 \mathrm{~mm}$ & $3 / 8^{\prime \prime}$ & $60-77$ & $70-88$ & 100 \\
\hline $4.75 \mathrm{~mm}$ & No. 4 & $43-59$ & $49-65$ & $65-87$ \\
\hline $2.00 \mathrm{~mm}$ & No. 10 & $29-45$ & $29-45$ & $43-61$ \\
\hline $0.425 \mathrm{~mm}$ & No. 40 & $14-25$ & $14-25$ & $16-29$ \\
\hline $0.180 \mathrm{~mm}$ & No. 80 & $8-17$ & $8-17$ & $9-19$ \\
\hline $0.075 \mathrm{~mm}$ & No. 200 & $4-8$ & $4-8$ & $5-10$ \\
\hline
\end{tabular}

Fuente: Elaboración propia. 
Tabla 3. Características generales del CA 60-70.

\begin{tabular}{llll}
\hline \multicolumn{1}{c}{ Ensayo } & \multicolumn{1}{c}{ Método } & \multicolumn{1}{c}{ Unidad } & CA 60-70 \\
\hline Ensayos sobre el CA original & & $0.1 \mathrm{~mm}$ & 65 \\
\hline Penetración $\left(25^{\circ} \mathrm{C}, 100 \mathrm{~g}, 5 \mathrm{~s}\right)$ & ASTM D-5 & - & $-0,7$ \\
\hline Índice de penetración & NLT 181/88 & Poises & 1750 \\
\hline Viscosidad absoluta $\left(60{ }^{\circ} \mathrm{C}\right)$ & ASTM D-4402 & $\mathrm{cm}$ & $>105$ \\
\hline Ductilidad $\left(25^{\circ} \mathrm{C}, 5 \mathrm{~cm} / \mathrm{min}\right)$ & ASTM D-113 & $\%$ & $>99$ \\
\hline Solubilidad en tricloroetileno & ASTM D-2042 & $\%$ & $<0,2$ \\
\hline Contenido de agua & ASTM D-95 & $\%$ & \\
\hline Punto de inflamación COC & ASTM D-92 & ${ }^{\circ} \mathrm{C}$ & \\
\hline Ensayos sobre el residuo del CA luego del RTFOT & & 0,4 \\
\hline Pérdida de masa & ASTM D-2872 & $\%$ & 70 \\
\hline Penetración $\left(25^{\circ} \mathrm{C}, 100 \mathrm{~g}, 5 \mathrm{~s}\right)$ & ASTM D-5 & $\%$ & \\
\hline
\end{tabular}

Fuente: Elaboración propia.

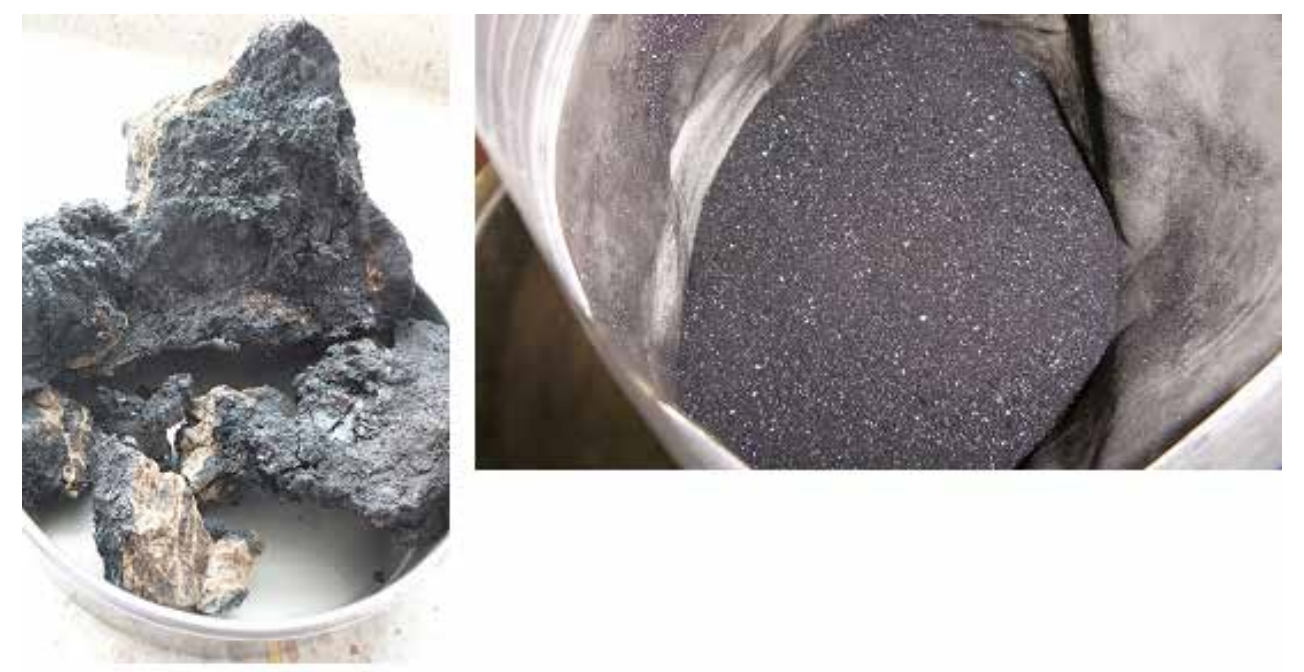

Figura 1. Gilsonita antes y después de trituración.

Fuente: Elaboración propia.

\section{Diseño de mezclas asfálticas}

Luego de realizar los ensayos al agregado pétreo y a los ligantes asfálticos se fabricaron cinco briquetas (compactadas a 75 golpes por cara) para cada porcentaje de asfalto de 4,5\%, 5,0\%, 5,5\%, 6,0 y $6,5 \%$, con el fin de efectuar el diseño Marshall para determinar el contenido óptimo de asfalto de las mezclas convencionales (sin gilsonita). Los porcentaje óptimos de cemento asfáltico fueron 5,0\%, $5,3 \%$ y $5,5 \%$ para el caso de las mezclas de tipos MDC-1, MDC-2 y MDC-3, respectivamente (ver las tablas 4-6). En estos porcentajes se están cumpliendo los requisitos mínimos exigidos por la especificación (INVIAS, 2007) para tránsitos de tipos NT1 y/o NT2 (bajos y medios volúmenes de tránsito). 
Tabla 4. Resultados del ensayo Marshall para diseño de mezcla MDC-1.

\begin{tabular}{ccccccc}
\hline CA [\%] & Estabilidad- $\boldsymbol{E}[\mathbf{k g}]$ & $\begin{array}{c}\text { Flujo- } \boldsymbol{F} \\
{[\mathbf{m m}]}\end{array}$ & $\boldsymbol{E} / \boldsymbol{F} \mathbf{[ k g / m m}]$ & $\begin{array}{c}\text { Densidad } \\
{\left[\mathbf{g} / \mathbf{c m}^{\mathbf{3}}\right]}\end{array}$ & $\begin{array}{c}\text { Vacíos } \\
{[\mathbf{\%}]}\end{array}$ & $\begin{array}{c}\text { Vacíos en AP } \\
{[\%]}\end{array}$ \\
\hline 4,5 & 1701,92 & 4,11 & 413,61 & 2,238 & 8,44 & 18,41 \\
\hline 5,0 & 1610,52 & 3,78 & 425,55 & 2,281 & 5,99 & 17,29 \\
\hline 5,5 & 1571,90 & 4,17 & 377,35 & 2,284 & 5,20 & 17,63 \\
\hline 6,0 & 1284,71 & 4,98 & 258,06 & 2,292 & 4,16 & 17,77 \\
\hline
\end{tabular}

Fuente: Elaboración propia.

Tabla 5. Resultados del ensayo Marshall para diseño de mezcla MDC-2.

\begin{tabular}{ccccccc}
\hline CA [\%] & Estabilidad- $\boldsymbol{[ \mathbf { k g } ]}$ & $\begin{array}{c}\text { Flujo-F } \\
{[\mathbf{m m}]}\end{array}$ & $\boldsymbol{E} / \boldsymbol{F} \mathbf{[ k g / m m ]}$ & $\begin{array}{c}\text { Densidad } \\
{\left[\mathbf{g} / \mathbf{c m}^{3}\right]}\end{array}$ & $\begin{array}{c}\text { Vacíos } \\
{[\% \mathbf{m}}\end{array}$ & $\begin{array}{c}\text { Vacíos en AP } \\
{[\%]}\end{array}$ \\
\hline 4,5 & 1396,54 & 3,73 & 374,03 & 2,257 & 7,66 & 17,71 \\
\hline 5,0 & 1500,60 & 3,73 & 401,90 & 2,288 & 5,72 & 17,05 \\
\hline 5,5 & 1551,87 & 3,78 & 410,05 & 2,295 & 4,71 & 17,21 \\
\hline 6,0 & 1235,97 & 4,72 & 261,61 & 2,300 & 3,80 & 17,47 \\
\hline
\end{tabular}

Fuente: Elaboración propia.

Tabla 6. Resultados del ensayo Marshall para diseño de mezcla MDC-3.

\begin{tabular}{cllllll}
\hline CA [\%] & Estabilidad- $\boldsymbol{E}[\mathbf{k g}]$ & $\begin{array}{c}\text { Flujo- } \boldsymbol{F} \\
{[\mathbf{m m}]}\end{array}$ & $\boldsymbol{E} / \boldsymbol{F} \mathbf{[ k g / m m}]$ & $\begin{array}{c}\text { Densidad } \\
{\left[\mathbf{g} / \mathbf{c m}^{3}\right]}\end{array}$ & $\begin{array}{c}\text { Vacíos } \\
{[\%]}\end{array}$ & $\begin{array}{c}\text { Vacíos en AP } \\
{[\%]}\end{array}$ \\
\hline 4,5 & 1214,60 & 3,91 & 310,51 & 2,279 & 6,78 & 16,93 \\
\hline 5,0 & 1278,11 & 3,78 & 337,71 & 2,316 & 4,56 & 16,02 \\
\hline 5,5 & 1321,77 & 3,91 & 337,91 & 2,311 & 4,08 & 16,66 \\
\hline 6,0 & 1176,73 & 4,88 & 241,29 & 2,307 & 3,51 & 17,22 \\
\hline
\end{tabular}

Fuente: elaboración propia

\section{Fase experimental}

Una vez se obtuvo el porcentaje óptimo de CA, se fabricaron nuevas briquetas agregando por vía seca la gilsonita a la mezcla asfáltica en relaciones de gilsonita y cemento asfáltico de $\mathrm{G} / \mathrm{CA}=5$, $10,15,20,25,30 \%$ (con respecto al peso total del cemento asfáltico). Por vía seca se entiende que el aditivo (la gilsonita en este caso) se adiciona a alta temperatura al agregado pétreo en el momento de la fabricación de la mezcla. Por cada porcentaje de aditivo se fabricaron cinco briquetas para ensayarlas en el aparato Marshall con el fin de evaluar la respuesta que experimentan las mezclas bajo carga monotónica. La temperatura de mezclado del CA con la gilsonita y el agregado pétreo fue $160{ }^{\circ} \mathrm{C}$. Se escogió esta temperatura debido a que por encima de la misma el asfalto experimenta 
envejecimiento por pérdida de componentes químicos por oxidación, y por debajo el mezclado se dificulta especialmente cuando el contenido de giIsonita es alto.

El ensayo que se ejecutó para evaluar la resistencia bajo carga monotónica de las mezclas convencionales y modificadas con gilsonita fue el Marshall (INV. E-748-07, AASHTO T 245-97).

\section{RESULTADOS}

En las figuras 2-4 se observa la evolución de la estabilidad $(E)$, la relación estabilidad - flujo $(E / F)$ y los vacíos con aire de la mezcla con el contenido de gilsonita $(\mathrm{G})$ adicionado a las mezclas de concreto asfáltico de tipos MDC-1, MDC-2 y MDC-3.

En la figura 2 se observa un incremento de $34,9 \%, 44,1 \%$ y $20,4 \%$ en la estabilidad cuando las mezclas MDC-1, MDC-2 y MDC-3, respectivamente, se modifican por vía seca con un contenido de $\mathrm{G}$ de $10 \%$ con respecto a la masa del CA. En ese mismo porcentaje de gilsonita y CA (G/ $\mathrm{CA}=10 \%$ ), las mezclas MDC-1, MDC-2 y MDC3 experimentan un incremento en su resistencia bajo carga monotónica (E/F) de 43,46\%, 41,8\% y $32,4 \%$, respectivamente (ver la figura 3 ). A partir de dicha relación $\mathrm{G} / \mathrm{CA}$, las mezclas experimentan un decaimiento en su resistencia $E / F$ debido en gran parte al incremento notable en los vacíos con aire en la mezclas (ver la figura 4).

Es interesante observar en la figura 4 que las mezclas experimentan el mayor incremento en rigidez y resistencia reportado con anterioridad cuando la relación $\mathrm{G} / \mathrm{CA}=10 \%$, aunque aumentan sus vacíos con aire. Para el caso de las mezclas MDC-1, MDC-2 y MDC-3, cuando la relación $\mathrm{G} / \mathrm{CA}=10 \%$, los vacíos con aire aumentan desde $5,99 \%$ hasta $7,93 \%, 5,0 \%$ a $6,66 \%$ y $4,08 \%$ a 4,94\%, respectivamente.

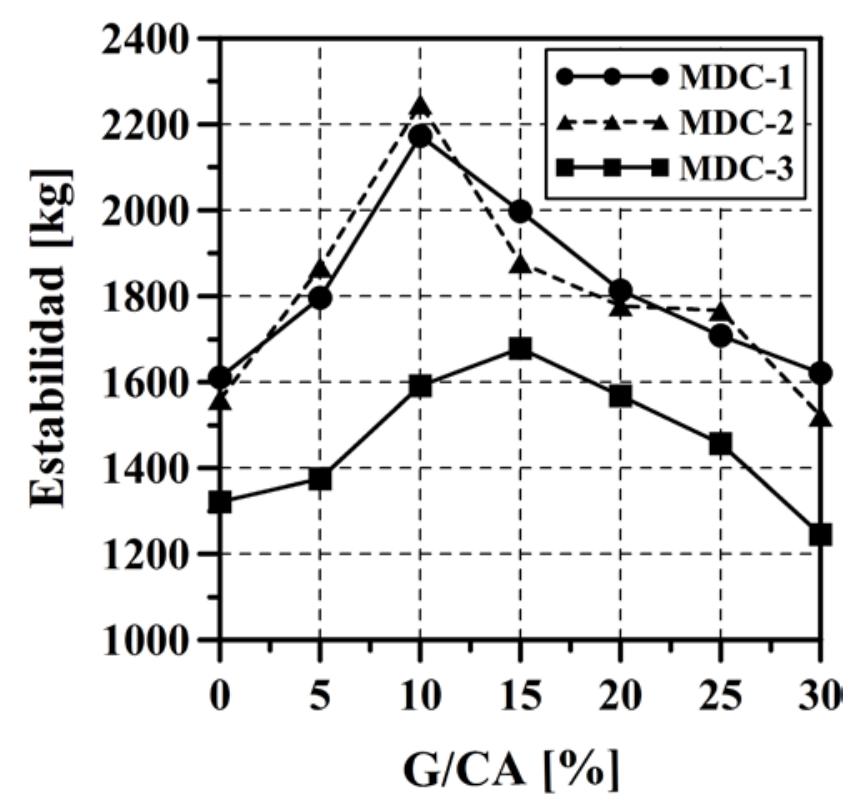

Figura 2. Evolución de la estabilidad con la relación G/ CA.

Fuente: Elaboración propia.

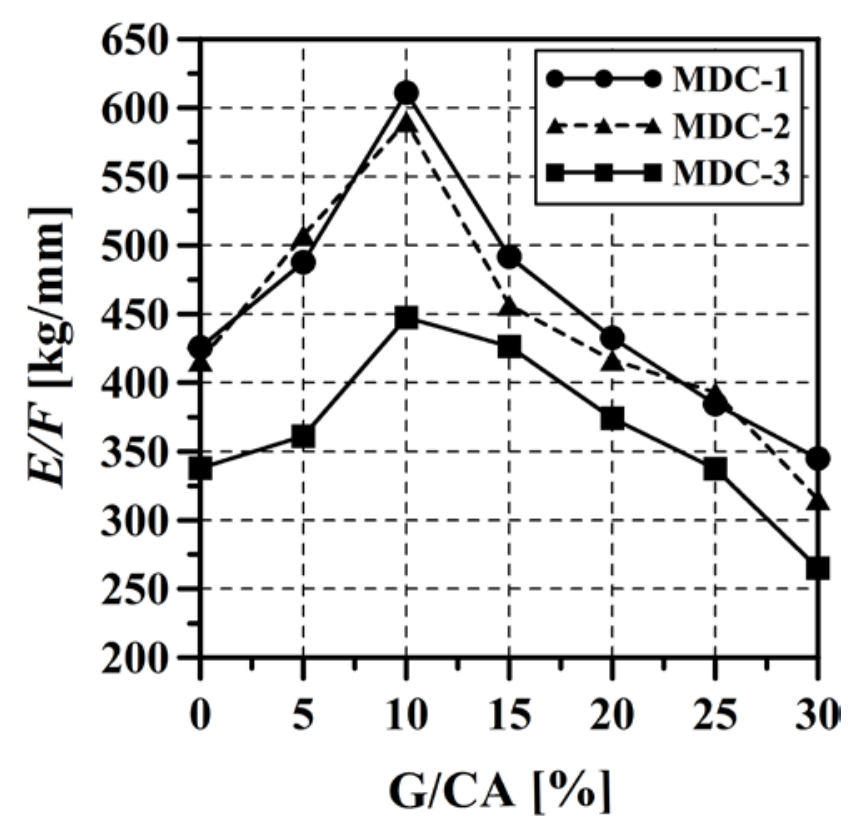

Figura 3. Evolución de la relación estabilidad/flujo con la relación $\mathrm{G} / \mathrm{CA}$.

Fuente: Elaboración propia. 


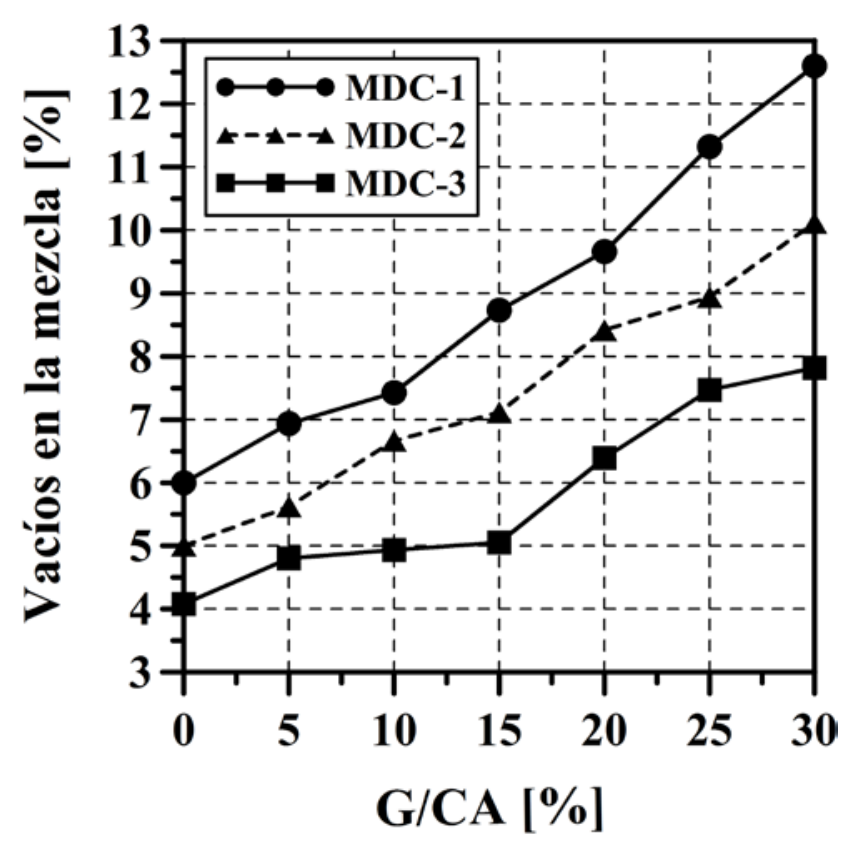

Figura 4. Evolución de los vacíos con aire en la mezcla con la relación G/CA.

Fuente: Elaboración propia.

\section{CONCLUSIONES}

El presente estudio midió la resistencia bajo carga monotónica que experimentan tres mezclas de concreto asfáltico cuando se modifican por vía seca con un asfalto natural de tipo gilsonita.

Se reporta un incremento entre $32,4 \%$ y $43,46 \%$ en dicha resistencia cuando se adiciona al agregado pétreo de las mezclas un contenido de gilsonita de $10 \%$ con respecto al peso del cemento asfáltico. Este incremento es mayor cuanto mayor sea el tamaño máximo de la partícula del agregado pétreo que conforma la mezcla asfáltica. Además se observa que dicho incremento lo experimentan las mezclas aunque el contenido de vacíos de las mismas aumenta.
El incremento en rigidez reportado en las mezclas asfálticas modificadas hace prever que la gilsonita como aditivo modificador de mezclas puede emplearse para mejorar la resistencia de las mismas a fenómenos como el ahuellamiento a altas temperaturas de servicio. Sin embargo, a bajas temperaturas la mezcla podría experimentar fragilización disminuyendo su resistencia a fenómenos como el agrietamiento por bajas temperaturas o la fatiga.

\section{FINANCIAMIENTO}

Universidad Distrital Francisco José de Caldas

\section{REFERENCIAS}

Esfeh, H. K., Ghanavati, B., \& GhaleGolabi, T. (2011). Properties of Modified Bitumen Obtained from Natural Bitumen by Adding Pyrolysis Fuel Oil. International Journal of Chemical Engineering and Applications, 2(3), 168-172.

INVIAS (1997). La Asfaltita como Modificador de los Asfaltos Colombianos. Modificación de Asfalto de la Refinería de Cartagena con Asfaltita. Bogotá, D.C., Colombia: Instituto Nacional de Vías.

INVIAS (2013). Especificaciones Generales de Construcción de Carreteras. Bogotá, D.C.: INVIAS.

INVIAS (2013a). Normas de Ensayos de Materiales para Carreteras. Bogotá, D.C.: Instituto Nacional de Vías.

Liu, J., \& Li, P. (2008). Experimental study on Gilsonite-modified asphalt. Airfield and Highway Pavements: Efficient Pavements Supporting Transportation's Future (pp. 222-228). Bellevue, Washington: ASCE.

Rondón, H. A., y Reyes, F. A. (2012). Evaluación de las propiedades mecánicas de una mezcla densa en caliente modificada con asfaltita. Revista de Ingeniería (36), 12-19.

Sagamone, P. (1995). Los asfaltos naturales colombianos. Bogotá D.C.: Instituto Nacional de Vías, Oficina de Investigaciones y Desarrollo Tecnológico. 
